\title{
Sistema embebido orientado a bebés en etapa de gateo para prevención de accidentes aplicado en un textil inteligente
}

\section{Embedded System Oriented to Babies in Crawl Phases for Accident Prevention in Applied a Smart Textile}

\author{
I. J. Chico-Morales ${ }^{1}$, S. K. Narváez-Pupiales ${ }^{1}$, A. C. Umaquinga-Criollo ${ }^{1}$, P. D. Rosero- \\ Montalvo ${ }^{1,2}$
}

\begin{abstract}
Resumen:
El gateo de los bebés es la forma de descubrir y aprender sus funciones motoras, cognitivas, sociales, emocionales, entre otras. Debido a esto, los infantes se exponen a diferentes peligros como: caídas, quemaduras, lesiones corporales entre las más comunes, donde el hogar es el lugar de mayores incidentes. Por lo antes expuesto, la presente investigación se enfoca en el desarrollo de un sistema embebido dentro de un textil inteligente que permita advertir y prevenir accidentes. El sistema se encuentra en una prenda de vestir en forma de arnés que cuenta con una conexión de rodilleras de gateo que incluye sensores magnéticos. Estos elementos son los encargados de poder detectar cintas de seguridad magnéticas colocadas previamente en lugares de mayor peligro en el hogar. Como resultado, el sistema emite una alerta con un tiempo de reacción de 7,6 segundos, después de ser activado el sistema.
\end{abstract}

Palabras clave: textil inteligente; sensor magnético; loT; accidentes de bebé; Arduino.

\begin{abstract}
:
The crawling of babies is the way to discover and learn their motor, cognitive, social, and emotional functions, among others. Because of this, infants are exposed to different dangers such as falls, burns, bodily injuries among the most common, where the home is the place of major incidents. Due to the above, the present investigation focuses on the development of an embedded system within an intelligent textile that allows accidents to be noticed. The system is in a garment in the form of a harness that has a connection with knee pads with magnetic sensors. These elements are responsible for detecting magnetic security tapes previously placed in places of greatest danger in the home. As a result, the system issues an alert with a reaction time of 7.6 seconds, after the system is activated.
\end{abstract}

Keywords: Textile intelligent; magnetic sensor; loT; baby crashes; Arduino.

\section{Introducción}

El gateo es el desplazamiento del bebé a través de una posición cuadrúpeda (García, Zúñiga, Ayala, \& Moreno, 2016). Este tipo de movimiento permite al bebé adquirir experiencia para la maduración motora (García et al., 2016) (Flehmig, 1988) (Trevino, 2008). Al ser una etapa de exploración y aprendizaje de conceptos espaciales, causan cambios a nivel perceptual, cognitivo, de lenguaje, social y emocional del infante (García et al., 2016). De esta manera, el bebé experimenta sus propios movimientos con el fin de iniciar su independencia (Flehmig, 1988). Según, (García et al., 2016) los bebés inician su etapa de gateo a los 5 meses, mientras que, (Oldak-Kovalsky y Oldak-Skvirsky,

\footnotetext{
1 Universidad Técnica del Norte, Ibarra, Ecuador (\{ijchico, sknarvaez, acumaquinga, pdrosero\}@utn.edu.ec).

2 Instituto Tecnológico Superior 17 de Julio, Yachay, Ecuador (prosero@istyachay.edu.ec).
} 
2015) (Babycenter, 2018) establecen como rango desde los 7 a 10 meses como su fase de inicio. Como consecuencia, a partir de esta etapa existe una mayor probabilidad de sufrir accidentes (Matute Seminario, Sarmiento Segovia, \& Torres Durán, 2015).

La Organización Mundial de la Salud (OMS) define como accidente: "acontecimiento fortuito, generalmente desgraciado o dañino, independiente de la voluntad humana, provocado por una fuerza exterior que actúa rápidamente y que se manifiesta por la aparición de lesiones orgánicas o trastornos mentales" (Minda Almagor, 2013), (Fuentes, 2013). De acuerdo con (Mizhquero y Fernanda, 2017) quien cita a (Minda Almagor, 2013) el 54\% de los percances en niños menores de 5 años se producen en la casa. Entre los accidentes más frecuentes se encuentran: ahogamiento, caídas, intoxicación, quemaduras, lesiones corporales, ingestas de cuerpos extraños, sustancias tóxicas (Minda Almagor, 2013), entre otros. La cocina, el cuarto de baño, el dormitorio, la sala de estar y el jardín son los lugares más propensos a sufrir percances (FACUA Andalucía, 2010), (Chico-Morales, Narváez-Pupiales, Umaquinga-Criollo y Rosero-Montalvo, 2017).

En la ciudad de Ibarra, Ecuador, en el Hospital San Vicente de Paúl en el año 2010 se registraron 5.166 casos de accidentes domésticos, de los cuales 494 correspondientes a niños y niñas menores de 5 años. De ellos, 422 casos en el rango de 1 a 4 años y 72 casos entre 0 y 1 año (Minda Almagor, 2013). Entre las atenciones y hospitalizaciones registradas fueron: fracturas, caídas, quemaduras e intoxicaciones mayormente del sexo masculino. Estos accidentes son muy comunes entre los niños, debido a su curiosidad de aprender sobre el mundo que los rodea. Debido a esto, es importante prevenir los riesgos para reducir este tipo de accidentes.

Generalmente en el entorno existe gran variedad de sistemas de seguridad electrónicos que principalmente se encargan de evitar o contrarrestar robos en el hogar, accidentes como incendios entre otros, pero exclusivamente enfocados a la preservación de la seguridad de los bebés en etapa de gateo, aún no es muy común, sin embargo en el mercado existen diferentes accesorios básicos que contribuyen al cuidado de un bebé en el hogar entre ellos: barreras de seguridad, tapas para los enchufes, protectores para puertas corredizas, entre otros pero que en muchos de los casos no son suficientes ante los numerosos peligros y carecen de otras funcionalidades complementarias.

$\mathrm{Si}$ se tiene como antecedente lo anteriormente expuesto el presente trabajo pretende ser de aporte para la prevención y reducción de accidentes en bebés en etapa de gateo, se propone el estudio, el desarrollo de un sistema electrónico de seguridad sobre la plataforma Arduino que alerta a los responsables del infante del peligro, activando una alarma que llega a un dispositivo móvil. Trabajos como (S. NúñezGodoy et al., 2016) (P. D. Rosero-Montalvo et al., 2017) (P. Rosero-Montalvo, 2017) presentan soluciones de sistemas electrónicos pero no orientados a bebés.

Esta investigación se organiza como sigue: en la Sección 2 se presentan los métodos y materiales aplicados en el presente estudio, la Sección 3 expone los resultados obtenidos; la Sección 4 los principales aspectos de análisis y discusión; mientras que, en la Sección 5 se presentan las conclusiones y recomendaciones de la presente investigación.

\section{Materiales y métodos}

En este apartado se describen los recursos tanto de hardware y software utilizados en el presente estudio así como el respectivo proceso de diseño y construcción del prototipo del sistema de seguridad para la prevención de accidentes de infantes en etapa de gateo.

Estos requerimientos son considerados para garantizar el funcionamiento $y$ eficiencia a la hora de salvaguardar la vida de los pequeños infantes, son como siguen: tiempo de reacción muy corto para la notificación inmediata del peligro; la cobertura del modem Wi-Fi a utilizar; el rendimiento adecuado de la fuente de alimentación, estético y 
de fácil colocación para no interferir con las actividades motrices del bebé además de funcionar como un sistema autónomo y sencillo de usar.

\subsection{Componentes de hardware}

La prenda inteligente constituye un mecanismo de seguridad para infantes menores de 18 meses y se encuentra constituido por los siguientes componentes (Chico-Morales, Narváez-Pupiales, Umaquinga-Criollo y Rosero-Montalvo, 2017):

- Placa de Control LilyPad Arduino: mantiene la comunicación con los sensores y en general asume el control de las funciones del sistema.

- Sensores Magnetic Reed: conectados a la placa de control y accionados una vez que se detecte la cercanía a las cintas magnéticas que constituyen las barreras a la salida a una zona de peligro.

- Cintas magnéticas: acopladas a los sensores Magnetic Reed, posee características semejantes a las de un imán siendo de esta forma ideal para cambiar el estado de los sensores mencionados cuando se encuentren cercanos a estas cintas.

- Módulo Wi-Fi ESP8266: permite la comunicación entre la red inalámbrica del hogar y la plataforma Internet de las cosas (del inglés Internet of Things, abreviado (IoT) para el envío de alertas, en caso de peligro del bebé. Se encuentra configurado en modo estación (STA), lo que permite la comunicación con cualquier dispositivo desde la Internet.

- Plataforma IoT: recibe y almacena la información proporcionada por el bloque de comunicación sobre todo los mensajes de alertas en caso de peligro. Se ha trabajado para el presente proyecto con la plataforma Open Source ThingSpeak (QuiñonezCuenca, González-Jaramillo, Torres y Jumbo, 2017), que facilita el almacenamiento en la nube además del análisis y visualización de componentes como Arduino, Rasperry, entre otros.

- Plataforma de Notificación IFTTT (If This Then That): que instalada en un smartphone en sistemas operativos Android y iOS facilita la comunicación con la plataforma IoT de acuerdo con las solicitudes recibidas y genera en caso de ser necesario o situaciones de peligro las notificaciones de alerta.

- Alimentación del Sistema: constituye una batería recargable que suministra la energía a todo el sistema, debidamente diseñada para proporcionar el funcionamiento del sistema por un período de 10 horas.

\subsection{Programación del sistema}

La programación de la placa de control utiliza el propio IDE (del inglés Integrated Development Environment) de Arduino y como lenguaje de programación el lenguaje C. La Figura 1 presenta el proceso que sigue el bloque de control para realizar las diferentes actividades para el funcionamiento del sistema, comenzando por la inicialización de las variables, librerías, comunicación serial y módulo ESP-01. En caso de existir un error de conexión en la red Wi-Fi del hogar el sistema notificará un error a través de la activación de un buzzer; caso contrario continúa con el proceso que envía una notificación y dato de inicio de los dispositivos y aplicaciones configuradas que permiten el escaneado del estado de los sensores Magnetic Reed, de forma que estos al detectar la cercanía con 
las cintas magnéticas inmediatamente por medio de la Plataforma loT alerta a los padres o responsables del cuidado del menor de un posible peligro.

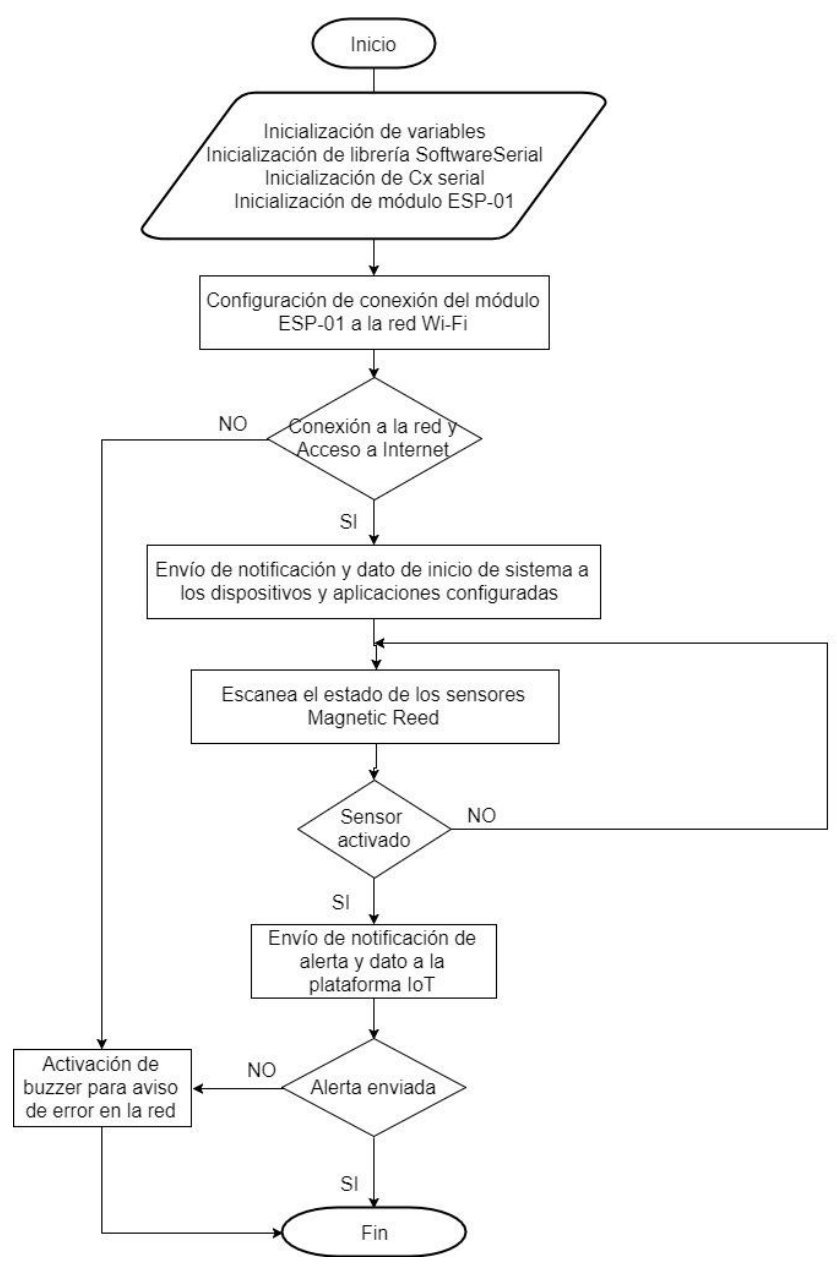

Figura 1. Procesos de la placa de control

\subsection{Construcción de la prenda inteligente}

Como antecedente importante para un correcto funcionamiento de la prenda inteligente es la definición de las zonas de peligro por parte de las personas responsables del cuidado del infante en etapa de gateo, ya que de esto depende la colocación de las cintas magnéticas, las cuales generan un activador al ser detectados por los sensores en caso que el bebé esté cerca de ellas. Los sensores son elementos que forman parte de las rodilleras que se intercomunican a la placa de control.

En cuanto a la comunicación de las alarmas de prevención, el sistema hace uso del módulo Wi-Fi a través de comunicación serial con la placa Arduino, permite al sistema formar parte de la red inalámbrica del hogar e Internet y mediante la cual los datos y alertas se almacenan en la web de loT que interactúa con la aplicación de notificaciones push instalada en el teléfono inteligente de los responsables del cuidado del infante. A continuación, se describe detalladamente el proceso de construcción de la prenda inteligente:

\section{- Circuito de conexión del sistema completo}

La Figura 2 muestra el esquema eléctrico de conexión de los bloques de control, sensores, comunicación y alimentación del sistema completo. 


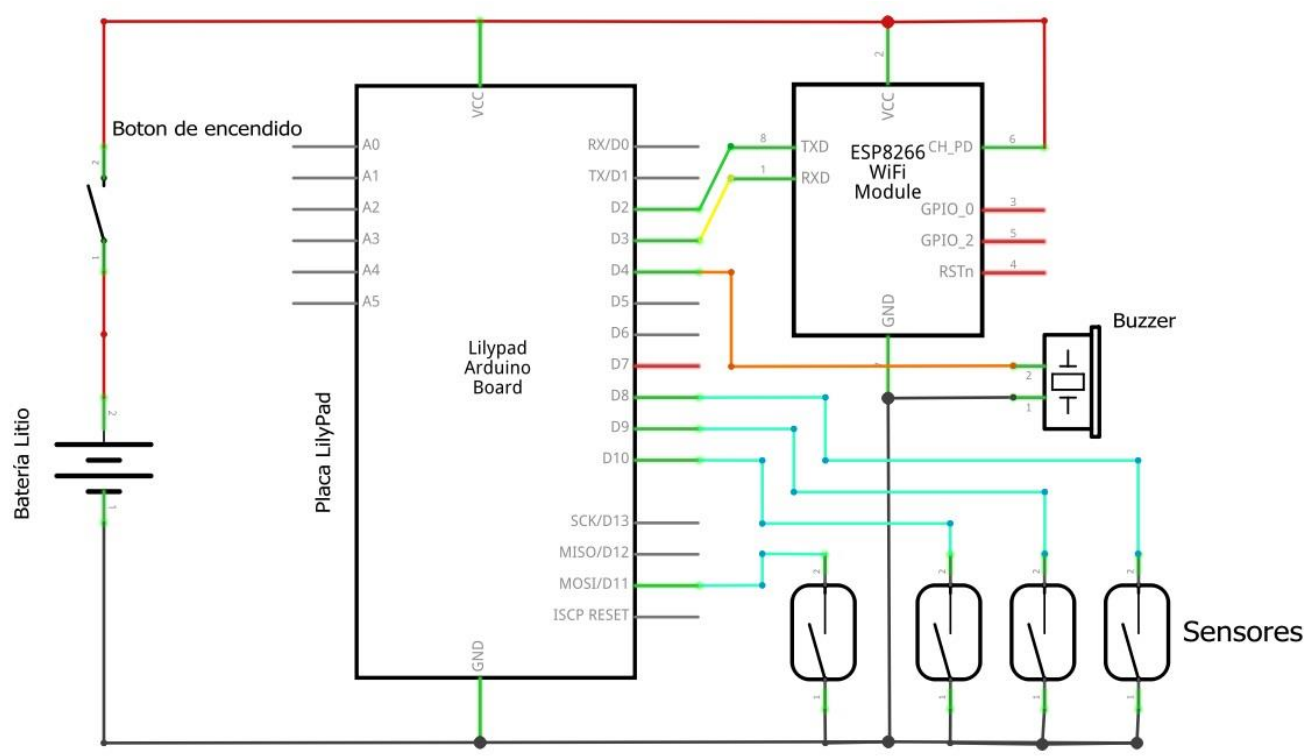

Figura 2. Esquema conexión de los componentes de hardware

\section{- Integración módulo Wi-Fi y módulo de alimentación}

Inicialmente se construye una placa mediante el software Eagle con pines semejantes a los de la placa LilyPad (Figura 3), con la finalidad que sean cosidos también en la tela, mediante hilo conductor. Los componentes de la nueva placa son: módulo Wi-Fi, buzzer, conector Micro-USB para carga, jumpers para la conexión de la batería, botón de enciendo, resistencias, LED indicadores de carga y diodo rectificador del circuito de carga. Los pines destinados a ser cosidos son: Rx y Tx del módulo Wi-Fi, la fuente de alimentación (positivo y negativo) y un pin del buzzer.

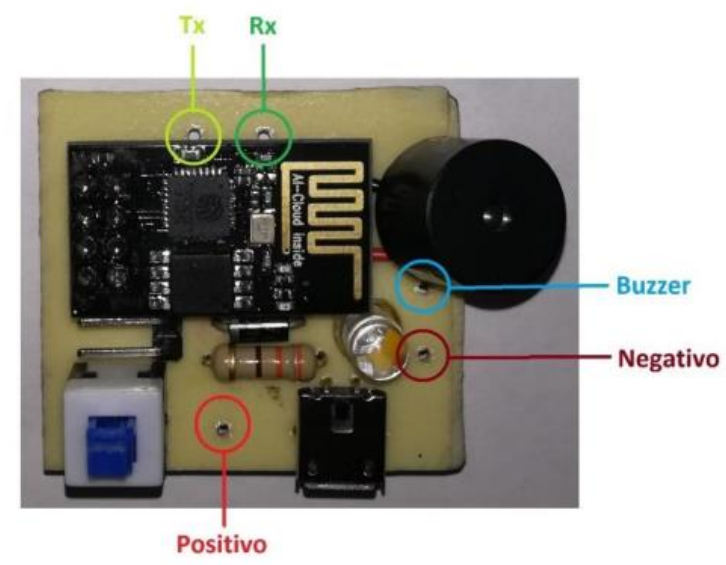

Figura 3. Placa de integración del Módulo Wi-Fi y alimentación

\section{- Integración de los componentes en tela}

Todo el hardware (segmento desmontable) se integra mediante broches de material conductor en una prenda de vestir tipo arnés de nylon con extensiones de hilo conductor a unas rodilleras de gateo donde se encuentran los sensores, que disparan las alarmas en caso de peligro. Además, cuenta con un bolsillo adicional el cual aloja la batería recargable detrás del segmento desmontable, es decir en la parte posterior de las placas (LilyPad y módulo Wi-Fi) como se muestra en la Figura 4. 


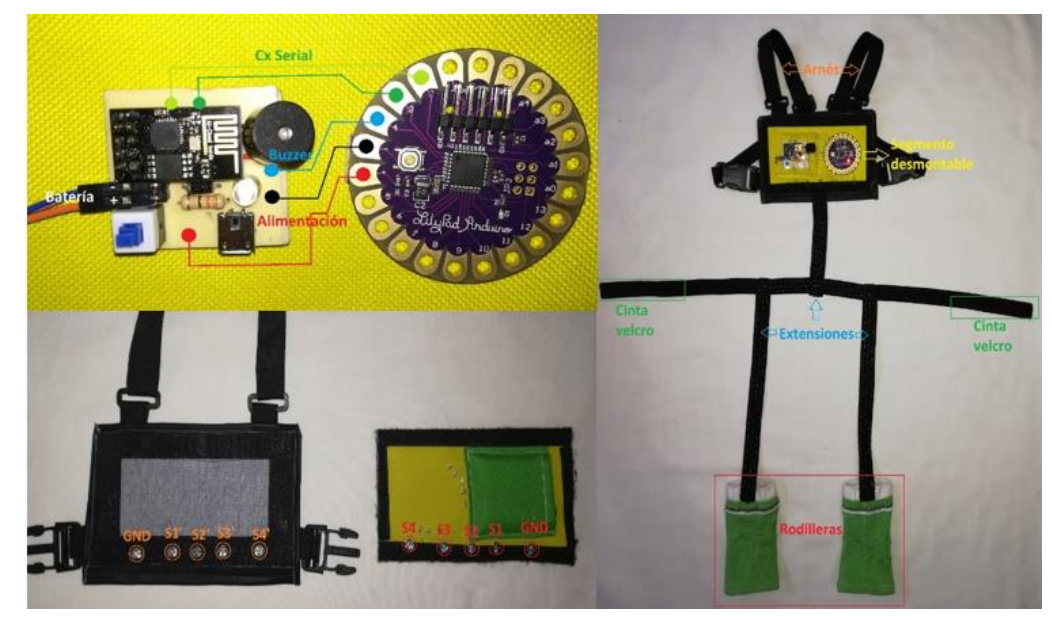

Figura 4. Integración de los componentes en una prenda de vestir tipo arnés

\section{- Instalación de los sensores}

Los sensores sujetos en las rodilleras de características elásticas se colocan en las zonas laterales de las piernas debajo de las rodillas debido a que estas áreas se encuentran a una distancia cercana a la superficie del suelo mientras el infante gatea, además esta área es propicia para evitar daños a los sensores mientras el bebé se desplaza.

\section{- Instalación de la cinta magnética}

Como se ha mencionado anteriormente las cintas magnéticas se colocan cercanas a las zonas de peligro a una distancia prudente con el objetivo de dar tiempo a los responsables del cuidado del bebé para reaccionar y acudir al sitio. Para determinar la distancia de ubicación de las cintas magnéticas de las zonas peligrosas se han utilizado los datos del Modelo dinámico para valoración del gateo (Hernández, Sánchez, Villanueva \& Pérez, 2016), de donde se puede obtener la velocidad promedio de gateo de los bebés en edades comprendidas entre 8 y 13 meses y es de $0,21 \mathrm{~m} / \mathrm{s}$. Además el tiempo promedio entre accionar los sensores y el establecimiento de la comunicación con la plataforma loT para el envío de las notificaciones es 7,36 segundos. Con estos datos la distancia de seguridad viene dada por la aplicación del principio $\mathrm{x}=\mathrm{v}^{\star} t$, la cual es $1,54 \mathrm{~m}$.

\section{- Almacenamiento de los datos en la plataforma web loT}

La plataforma loT a utilizar es ThingSpeak, la cual requiere la creación de una cuenta para su habilitación desde su sitio oficial. Esta plataforma al trabajar con el sistema de seguridad recibirá un dato y lo almacenará cada vez que se active un sensor (Alvear, 2017). El envío de datos es a través de peticiones HTTP al módulo ESP-01 (módulo Wi-Fi). La información a recibir podría ser el valor 100 indicando que se ha activado una alarma y un valor de cero así como el inicio del sistema.

\section{- Notificaciones}

Corresponde al mecanismo de advertencias cuando el infante esté cerca de las zonas de riesgos, a través de alertas al Smartphone de los responsables del cuidado. La aplicación utilizada en el móvil IFTTT funciona a través de la vinculación con la plataforma loT. Para acceder a los servicios de IFTTT es necesario registrarse desde la página oficial o incluso desde la aplicación. 


\section{Resultados}

En el proceso de implementación se realizaron las pruebas respectivas para verificar su funcionamiento, entre ellas la distancia de seguridad mínima para la instalación de las cintas magnéticas es de $1,54 \mathrm{~m}$ de las zonas consideradas como peligrosas.

Las pruebas de inicio del sistema con una red de 2 Mbps de velocidad tiene un tiempo promedio de inicio del sistema 18.7 segundos, como tiempo estimado de notificación de alerta 7.49 segundos tomados con cintas instaladas en diferentes distancias: 4 metros del router (escalera), otro a 2 metros (baño) y el último a 3 metros (cocina), a mayores distancias, se han probado exitosamente envíos de notificaciones a 13 metros en un tiempo entre 6,8 a 7,8 segundos. La notificación que se envía al activarse un sensor tiene como texto "Alerta! Tu bebé está en peligro".

El tiempo de duración de la batería es de 12 horas con 10 minutos, se recomienda cargar la batería 1 hora antes de usarlo.

Los datos son recopilados en la plataforma ThingSpeak, el sistema envía un 0 (cero) cuando se inicia el sistema y 100 (cien) como indicador de que se ha activado una alarma que comunica inmediatamente al móvil de los responsables del cuidado del bebé a través de la plataforma IFTTT.

\section{Discusión}

El sistema inteligente ha sido evaluado dentro de un ambiente controlado y, posteriormente en una situación real, de lo cual se obtuvo que el tiempo promedio de respuesta es de 7,36 segundos a una distancia de $1,54 \mathrm{~m}$ de distancia de las zonas peligrosas, tiempo en primera instancia considerado como prudente para que los encargados del cuidado del bebé puedan acudir al sitio y socorrer al infante. Cabe indicar que el tiempo promedio de respuesta del sistema está condicionado a la velocidad de la conexión a Internet, de la distancia al punto de acceso y calidad de la señal inalámbrica.

Con respecto a la posibilidad de afectar este sistema a las actividades diarias y normales del niño, se ha pensado durante la construcción en un sistema flexible formado de un arnés tipo ajustable a la estura promedio de un infante de entre 6 y 18 meses de edad, lo cual minimiza la posibilidad de incidir en las capacidades motrices del infante.

Como futuros trabajos se espera que el sistema pueda ampliar su rango de cobertura mediante plataformas de machine to machine. De esta forma será más flexible con comunicación con antenas de comunicación móvil.

\section{Conclusiones y recomendaciones}

El gateo es una de las etapas de mayor importancia para el desarrollo de los bebés, le permite explorar y desarrollar capacidades motoras, de equilibro, tacto, entre otras, de forma que es inevitable limitar a los infantes prescindir de este mecanismo de desplazamiento entre los 8 y 13 meses de edad. Sin embargo dentro del hogar pueden existir ambientes o lugares peligrosos como las escaleras, baño, cocina, entre otros que pueden convertirse en un peligro, de manera que la presente investigación pretende contribuir a la disminución de la cantidad de accidentes de bebés mediante la alerta y notificación que el menor se está acercando a una zona definida como riesgosa, con la ayuda de herramientas tecnológicas tanto de software y de hardware que combina un sistema embebido con textiles, plataformas loT y de notificación.

Los componentes utilizados tanto de hardware como de software fueron seleccionados de un conjunto de herramientas similares, de forma que la construcción del sistema requirió previamente la evaluación y comparativa para garantizar el funcionamiento y eficiencia del mismo. Sin embargo para nuevos trabajos se recomienda 
optimizar el consumo de la batería y mejorar los tiempos de reacción y comunicación del sistema.

\section{Bibliografía}

Alvear V., Rosero P., Peluffo D., Pijal J. (2016). Internet de las Cosas y Visión Artificial, Funcionamiento y Aplicaciones: Revisión de Literatura. Vol. 8 Núm. 1: Edición Especial INCISCOS. https://doi.org/10.29019/enfoqueute.v8n1.121

Babycenter. (2018). Gatear - BabyCenter. Recuperado el 11 de febrero de 2018, a partir de https://espanol.babycenter.com/a900442/gatear

Chico-Morales, I. J., Narváez-Pupiales, S. K., Umaquinga-Criollo, A. C. y RoseroMontalvo P.D. (2017). Textil Inteligente de Prevención para Bebés en Etapa de Gateo. Wearable textil for accident prevention for babies in crawl phase. In Proceedings of the 2nd International Conference on Information Systems and Computer Science, Quito, Ecuador.

FACUA Andalucía. (2010). FACUA-Consumidores en Acción. Recuperado el 12 de febrero de 2018, a partir de http://www.facua.org/es/guia.php?/d=132

Flehmig, I. (1988). Desarrollo normal del lactante y sus desviaciones: diagnóstico y tratamiento tempranos. Editorial Médica Panamericana.

Fuentes, F. J. C. (2013). Apoyo al soporte vital avanzado. SANT0108. IC Editorial.

García, M. H., Zúñiga, M. E. S., Ayala, D. V., \& Moreno, J. C. P. (2016). Modelo dinámico para valoración del gateo. Rev Mex Med Fis Rehab, 28(1-2), 28-32.

Godoy S., Alvear V., Realpe S., Rosero P. (2016). Human-sitting-pose detection using data classification and dimensionality reduction,"IEEE Ecuador Technical Chapters Meeting (ETCM), Guayaquil, 2016, pp. 1-5.

Matute Seminario, A. M., Sarmiento Segovia, M. J., \& Torres Durán, A. E. (2015). Conocimientos, actitudes y prácticas sobre el desarrollo psicomotor dirigido a madres de niños y niñas menores de un año de edad que asisten a la Fundación Pablo Jaramillo Crespo. Recuperado a partir de http://dspace.ucuenca.edu.ec/handle/123456789/23069

Minda Almagor, A. C. (2013). Factores influyentes asociados en la aparición de accidentes domésticos en niñas y niños menores de 5 años, atendidos en el Servicio de Emergencia del Hospital San Vicente de Paúl, de la Ciudad de Ibarra en el período noviembre 2010 a julio del 2011.

Mizhquero, N., \& Fernanda, Y. (2017). Determinación de la prevalencia de accidentes domésticos en pacientes menores de 18 años atendidos por el personal de atención prehospitalaria del Cuerpo de Bomberos Quito en el período enero-diciembre 2016 (B.S. thesis). Quito: UCE.

Oldak-Kovalsky, B., \& Oldak-Skvirsky, D. (2015). Gateo. Revisión de la literatura médica. Revista Mexicana de Pediatría, 144-148.

OMS. (2016, septiembre). OMS | Reducción de la mortalidad en la niñez. Recuperado el 12 de febrero de 2018, a partir de

http://www. who.int/mediacentre/factsheets/fs178/es/

Rosero, P., Peluffo, D., Godoy, P. (2017). Elderly fall detection using data classification on a portable embedded system," IEEE Second Ecuador Technical Chapters Meeting (ETCM), Salinas, pp. 1-4. doi: 10.1109/ETCM.2017.8247529

Rosero-Montalvo, P., Jaramillo, D., Flores, S., Peluffo, D., Alvear, V., Lopez, M. (2017). "Human Sit Down Position Detection Using Data Classification and Dimensionality Reduction", Advances in Science, Technology and Engineering Systems Journal, vol. 2, no. 3, pp. 749-754.

Quiñonez-Cuenca, M., González-Jaramillo, V., Torres, R. y Jumbo, M. (2017). Sistema de Monitoreo de Variables Medioambientales usando una Red de Sensores 
Inalámbricos y Plataformas de Internet de las Cosas. Enfoque UTE. Recuperado a partir de http://ingenieria.ute.edu.ec/enfoqueute/index.php/revista/article/view/139

Trevino, C. M. J. (2008). Neurofacilitación / Neurofacilitation: Técnicas de rehabilitación neurológica / Neurological rehabilitation techniques (Edición: Reissue). México: Editorial Trillas Sa De Cv. 